\title{
UPTAKE AND EGESTION OF POLYHYDROXYALKANOATE MICROBEADS IN THE MARINE COPEPOD Nitokra lacustris pacifica
}

\author{
TAN SUET MAY AMELIA ${ }^{1}$, SITI NOORHUDA FATIHAH SUKRI ${ }^{1}$, ANNISA NURSABRINA \\ JAAPAR $^{2}$, ROSWATI MD AMIN ${ }^{1,3}$ AND KESAVEN BHUBALAN*1,3,4,5 \\ ${ }^{1}$ Faculty of Science and Marine Environment, ${ }^{2}$ Institute of Oceanography and Environment, ${ }^{3}$ Ocean Polution and \\ Ecotoxicology Research Group, ${ }^{4}$ Institute of Marine Biotechnology, Universiti Malaysia Terengganu, 21030 Kuala Nerus, \\ Terengganu, Malaysia, ${ }^{5}$ Malaysian Institute of Pharmaceuticals and Nutraceuticals, NIBM, 11700 Pulau Pinang, Malaysia.
}

*Corresponding author: kesaven@umt.edu.my

Submitted: 13 September 2019 Accepted: 10 April $2020 \quad$ http://doi.org/10.46754/jssm.2020.06.005

\begin{abstract}
Petrochemical-based microplastics are widely distributed and abundant in the marine environment, and they have become a global economic and environmental concern. Microplastics are mistakenly ingested by zooplankton as food, subsequently disrupting the biological process of zooplankton, a crucial food source for many secondary consumers. Research has led to the development of biodegradable plastics, such as polyhydroxyalkanoate (PHA), to remediate this problem. This study aims to evaluate the ingestion and egestion of PHA microbeads by the Nitokra lacustris pacifica copepod. The polymer $\mathrm{P}(3 \mathrm{HB})$ produced from Massilia haematophila UMTKB-2 was used to develop PHA microbeads. The PHA microbeads were then added to copepod cultures to study the ingestion of the PHA microbeads and its effects on the copepods. The copepods were viewed under an inverted fluorescence microscope to confirm the presence of the PHA microbeads in their digestive tracts and faecal pellets. The percentage of PHA microbeads ingested by the copepods and their incorporation in faecal pellets were higher, $>70 \%$ and $>80 \%$, respectively, after being exposed for $24 \mathrm{~h}$ and three days were required to fully egest the microbeads. However, no mortality was observed in this short-term incubation experiment.
\end{abstract}

Keywords: Polyhydroxyalkanoate, microplastics, zooplankton

\section{Introduction}

The application and discharge of plastics or plastic-containing waste pose a threat to wildlife and pollute the environment as they decompose very slowly, eventually accumulating in the ultimate sink for plastic waste - the ocean. Petrochemical-based plastics have been widely used in numerous consumer products for daily use, as well as for household, packaging, cosmetic, medical and agricultural purposes. The forms of petrochemical-based plastics include microplastics found in most commercial products for personal hygiene and domestic cleaning, such as toothpastes, scrubs, shampoos, soaps, facial cleansers, and abrasive cleaning agents. Microplastics are among the chief plastic waste in the ocean, leading to marine pollution. They are typically made of polyvinyl chloride, nylon, polyethylene terephthalate, polyethylene, polypropylene, polystyrene, polyvinyl alcohol, and polyamide (Napper et al., 2015; Avio et al., 2016; Carr et al., 2016).

Microplastic pollution is a rising global concern due to its distribution, persistence, and adverse effects to the ecosystem. The concentration of microplastics reached up to 2500 particles $\mathrm{km}^{-2}$ in global marine regions, including the Arctic region, Northwest Atlantic, Northeast Atlantic Ocean, Southern North Sea, Mediterranean Sea, and the East China Sea (Auta et al., 2017). Microplastics have not only polluted multiple geographical regions, they also persist as petrochemical-based microplastics are continuously used in various products for functionality. Microplastics also act as a carrier that adsorbs and bioaccumulates environmental pollutants within living organisms, thereby increasing the bioavailability of pollutants that are eventually biomagnified in the tissues of organisms at higher levels of the food chain, 
such as humans. The global environmental concern regarding plastic waste has diverted attention towards the development of bio-based biodegradable plastics.

Previous reports have revealed microplastic ingestion by zooplankton (Md Amin et al., 2020) whereby they are capable of varied microplastic uptake depending on the size of the microplastics, the life stage of the copepod and the species of the copepod. Microplastics are ingested by zooplankton through filter-feeding and egested in faecal pellets, generally within several hours (Barnes et al., 2009; Cole et al., 2013). It is imperative to study the ingestion and egestion of microplastics by planktonic organisms as they are at the base of the food web, hence they could bio-magnify chemical contaminants associated with microplastics to subsequent trophic levels (Turner, 2004). The types of zooplankton reported to ingest microplastics include holoplankton (Copepoda, Tunicata, and Euphausiacea), meroplankton (Mollusca and Decapoda), and microzooplankton (Dinoflagellata) (Cole et al., 2013).

Copepods are among the most studied organisms related to the environmental impacts of microplastics (Cole et al., 2013; Cole \& Galloway, 2015). As the effects have been shown to be species-specific, there has not been any previous study regarding the ingestion of microplastics by the copepod Nitokra lacustris pacifica. However, Nitocra spinipes, a copepod from a closely related genus, was screened for acute toxicity of leachate from weathering plastics. Leachate from 8 out of 21 types of plastics induced acute toxicity and mortality in $N$. spinipes before and/or after irradiation (Bejgarn et al., 2015). A proper study on the feeding preferences of $N$. lacustris pacifica has not been reported. Furthermore, the size of conventional microplastics typically ingested by copepods are between $10 \mu \mathrm{m}$ and $20 \mu \mathrm{m}$ for polyethylene (Coppock et al., 2019). The common types of microplastics ingested by copepods or used in copepod ingestion studies are polyethylene terephthalate, nylon, and polyethylene (Coppock et al., 2019).
Polyhydroxyalkanoate (PHA) is a microbially synthesised, biodegradable, and biocompatible polymer with thermoplastic or elastomeric properties (Amirul et al., 2008). The PHA material is chiefly produced by certain genera of bacteria fermented in low-nutrienthigh-carbon conditions with applications in packaging, medical or pharmaceutical industries (Verlinden et al., 2007). PHA types that exhibit different thermal and mechanical properties can be altered for specific applications by manipulating the strain, carbon source, growth conditions and the PHA synthase gene (Ntaikou et al., 2018). Among the types of PHA, poly(3-hydroxybutyrate) $[\mathrm{P}(3 \mathrm{HB})]$ is the most common and naturally occurring PHA, which is more biocompatible and easily processable than other PHA types. $\mathrm{P}(3 \mathrm{HB})$ also has mechanical properties desirable for medical and pharmaceutical applications, such as the pericardial patch, cardiovascular stent and drug delivery (Williams \& Martin, 2002). The increasing applications and commercial use of PHA require extensive research concerning the environmental impact of PHA-based products. Discarded PHA products also degrade into microplastics eventually. There are very few reports on PHA microplastics in the marine environment or the feeding of PHA microplastics to copepods. In this study, the PHA microbeads developed using $\mathrm{P}(3 \mathrm{HB})$ were characterised and the effects of ingestion and egestion of the PHA microbeads by the harpacticoid copepod $N$. lacustris pacifica were evaluated.

\section{Materials and Methods}

\section{Biosynthesis of $\mathrm{P}(3 \mathrm{HB})$}

$\mathrm{P}(3 \mathrm{HB})$ was produced by the bacterial strain Massilia haematophila UMTKB-2 (Genbank accession number KT025845) isolated from brackish water in Mengabang Telipot, Kuala Nerus, Terengganu, Malaysia (Kiun et al., 2016). The strain subculture, glycerol stock, seed culture, nutrient rich (NR) medium, mineral salt medium (MSM) and trace elements were prepared, and then $\mathrm{P}(3 \mathrm{HB})$ was produced according to the method done by Kiun and co- 
workers (Kiun et al., 2019). The cell biomass was obtained by measuring the optical density of the culture broth at a wavelength of $600 \mathrm{~nm}$. Subsequently, the cell culture was centrifuged twice at $4^{\circ} \mathrm{C}$ and $9,000 \mathrm{rpm}$ for $10 \mathrm{~min}$. The cell pellet was stored at $-80^{\circ} \mathrm{C}$ overnight before being freeze-dried at $-45^{\circ} \mathrm{C}$ for $72 \mathrm{~h}$ using a freeze-dry machine (Labconco, USA). Gas chromatography was carried out to determine the composition of the PHA produced (Yatim et al., 2017).

\section{Extraction of $\mathrm{P}(3 \mathrm{HB})$}

The polymer $\mathrm{P}(3 \mathrm{HB})$ was extracted (Figure 1) as previously described by Kiun and coworkers (Kiun et al., 2019). Freeze-dried cells were stirred with chloroform $\left(\mathrm{CHCl}_{3}\right)$ for $48 \mathrm{~h}$ to disrupt the cells and extract $\mathrm{P}(3 \mathrm{HB})$ (Amirul et al., 2008). The inactivation and removal of endotoxin were achieved by using hydrogen peroxide, after which the endotoxin level was tested using E-TOXATETM Kits (Sigma Aldrich, USA) (Rao et al., 2010; Govindasamy et al., 2019).

\section{The Development of PHA Microbeads}

The development of PHA microparticles in the form of microbeads (Figure 1) was carried out using the double emulsion solvent evaporation technique as described by Govindasamy and co-workers (Govindasamy et al., 2019). The microbeads were extracted using the suction filtration technique with $0.2-\mu \mathrm{m}$ nylon-66 filter papers, and then washed three times with distilled water and dried overnight for scanning electron microscope analysis (Govindasamy et al., 2019).

\section{In-vivo Evaluation of PHA Microparticle on Copepod}

The copepod $N$. lacustris pacifica was cultured and maintained in the laboratory under $12 \mathrm{~h}$ light and $12 \mathrm{~h}$ dark cycle feed with microalgae Nannochloropsis sp. The first generation of adult $N$. lacustris pacifica was sorted and left unfed in filtered seawater for $24 \mathrm{~h}$. The copepods were then subjected to two different treatments; fluorescent PHA microbeads (dyed with Nile $\operatorname{Red}(1 \mathrm{mg} / \mathrm{mL}$ in acetone $)$ ) with a concentration of 700 PHA microbead $\mathrm{mL}^{-1}$ prepared in i) filtered seawater and ii) unfiltered seawater. This selected concentration of microbeads was in the scope of a laboratory-based study without exceeding the extreme concentration used in ecotoxicological studies (Lee et al., 2013; Cole et al., 2015). Filtered seawater was screened with Whatman GF/F filter membranes (47 $\mathrm{mm}$ diameter; $0.7 \mu \mathrm{m}$ pore size) to remove suspended particle while unfiltered seawater was produced by filtering through a $20-\mu \mathrm{m}$ mesh net to remove any zooplankton. These were conducted to determine if the availability of suspended particle will significantly affect the PHA ingestion of the copepods. Both treatments were further added with $300 \mu \mathrm{g} \mathrm{C}$ $\mathrm{L}^{-1}$ of Nannochloropsis sp. Live copepods were individually sorted and placed in $5.5-\mathrm{cm}$ petri dishes (10 individual per replicate; 9 replicates per treatment) filled with $10 \mathrm{~mL}$ of the above treatment.

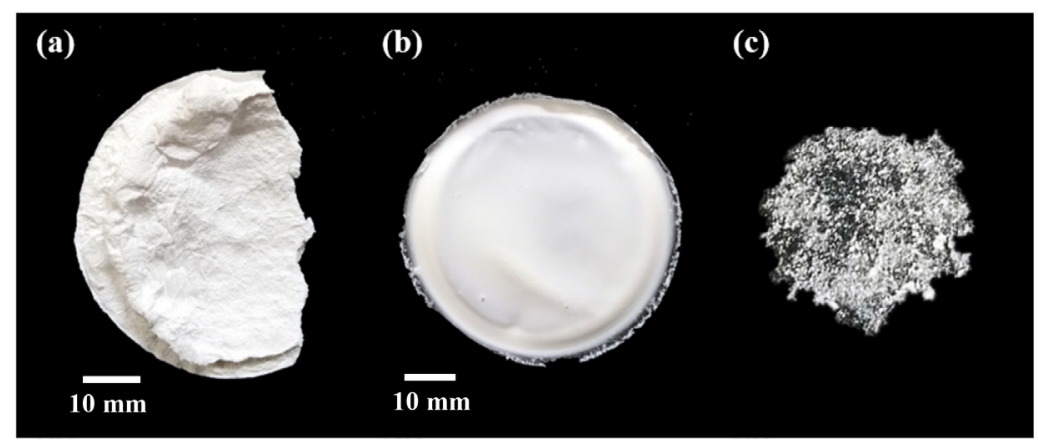

Figure 1: (a) Crude $\mathrm{P}(3 \mathrm{HB})$ extracted from $M$. haematophila UMTKB-2, (b) PHA in the form of $\mathrm{P}(3 \mathrm{HB})$ cast film and (c) PHA microbeads developed using the double emulsion solvent evaporation technique 
To test the microbead ingestion, copepods were first exposed for $24 \mathrm{~h}$ with the respective treatment. To see the differences in egestion time after ingestion and the presence of microbeads in faecal pellets, the copepods were removed from the initial treatment and transferred to a petri dish filled with filtered seawater. About 5 drops of formalin were added into the petri dish to euthanise the copepods prior to the observation. The experiment was stopped when no PHA microbeads were detected in their bodies and faecal pellets. The egestion time and microplastic incorporation in the pellets were then calculated based on the percentage of the microbead presence over the total individual/ pellets tested by taking 3 replicates $(n=30)$ per treatment every $24 \mathrm{~h}$ for further observation (Lee et al., 2013; Cole et al., 2015). The observations on the microbeads in both body and faecal pellets were done using the IX81 Inverted Fluorescence Microscope (Olympus, Japan). Statistical analysis was run using the software IBM SPSS Statistics 23.0. The Kruskal-Wallis test was used to compare the percentage of PHA microbeads ingested with the faecal pellets between filtered and unfiltered treatments.

\section{Results and Discussion}

\section{The Characterisation of $\mathrm{PHA}$ and $\mathrm{PHA}$ Microbeads}

M. haematophila UMTKB-2 produced 0.744 $\mathrm{g} / \mathrm{L}$ of $\mathrm{P}(3 \mathrm{HB})$ using a carbon:nitrogen $(\mathrm{C} / \mathrm{N})$ ratio of 50 and glucose as the sole carbon source. The endotoxin level of the PHA microbeads after the removal of the former was below the minimum safety threshold. Strain UMTKB-2 was observed to produce a higher $\mathrm{P}(3 \mathrm{HB})$ yield than other species of the same genus. A glucoseutilising seaweed isolate, Massilia sp. UMI-21, utilised a $\mathrm{C} / \mathrm{N}$ ratio of 60 to produce $\mathrm{P}(3 \mathrm{HB})$ with a concentration of $0.02 \pm 0.01 \mathrm{~g} / \mathrm{L}$, which was less than the $\mathrm{P}(3 \mathrm{HB})$ produced by strain UMTKB-2 despite using a higher $\mathrm{C} / \mathrm{N}$ ratio (Han et al., 2014). Among other seven PHA-producing bacteria of the Massilia genus, which were Massilia albidiflava, Massilia lutea, Massilia dura, Massilia plicata, Massilia brevitalea,
Massilia aerilata and Massilia aurea, the highest $\mathrm{P}(3 \mathrm{HB})$ concentration obtained was $1.25 \mathrm{~g} / \mathrm{L}$ from $M$. lutea using $4 \mathrm{~g} / \mathrm{L}$ of soluble starch as the sole carbon source (Cerrone et al., 2011).

Microbeads are particulates commonly suspended in surfactants to mechanically exfoliate and cleanse the face or body. Microbeads are manually massaged into the skin to dislodge shedding outer epidermis, improve skin tactile softness and visual smoothness, as well as promote the absorption and application of cosmeceutical or skincare products (Draelos, 2019). The PHA microbeads developed were spherical-shaped and possessed a heterogenous average size range of $10.1 \mu \mathrm{m}$ to $140 \mu \mathrm{m}$ with an average diameter of $38.44 \mu \mathrm{m}$ (Figure 2). The size of commercial exfoliating microbeads made from petrochemical-based plastic ranges from 10 to $500 \mu \mathrm{m}$, while the size of human skin pores ranges from 250 to $500 \mu \mathrm{m}$ (Flament et al., 2015; Napper et al., 2015). Furthermore, according to reports on Japanese coastal waters, the typical size of microbeads found in the environment was less than $2 \mathrm{~mm}$, whereby microplastics between $300 \mu \mathrm{m}$ and $400 \mu \mathrm{m}$ in size were dominantly microbeads. Additionally, $10 \%$ of the microplastics were microbeads (smaller than $800 \mu \mathrm{m}$ ) (Isobe, 2016; Tanaka \& Takada, 2016). The size of the PHA microbeads produced was more compatible with the size of human skin pores than the sizes of the PHA microbeads reported by Mohamed and co-workers that ranged from $0.3 \mu \mathrm{m}$ to $0.6 \mu \mathrm{m}$ (Mohamed et al., 2017), and those of Murueva and co-workers that ranged from $0.7 \mu \mathrm{m}$ to 2.6 $\mu \mathrm{m}$ (Murueva et al., 2013). The heterogenous size of the PHA microbeads supplied to the microbead treatment setup is more akin to the natural seawater environment than the size of controlled microbeads.

The material $\mathrm{P}(3 \mathrm{HB})$ was the chosen $\mathrm{PHA}$ type used to produce the microbeads in this study as $\mathrm{P}(3 \mathrm{HB})$ is the most commonly found and studied polymer of the PHA family (Yatim et al., 2017; Amelia et al., 2019). Further study of the ingestion and egestion of other types of PHA by plankton in different environmental conditions is recommended. 

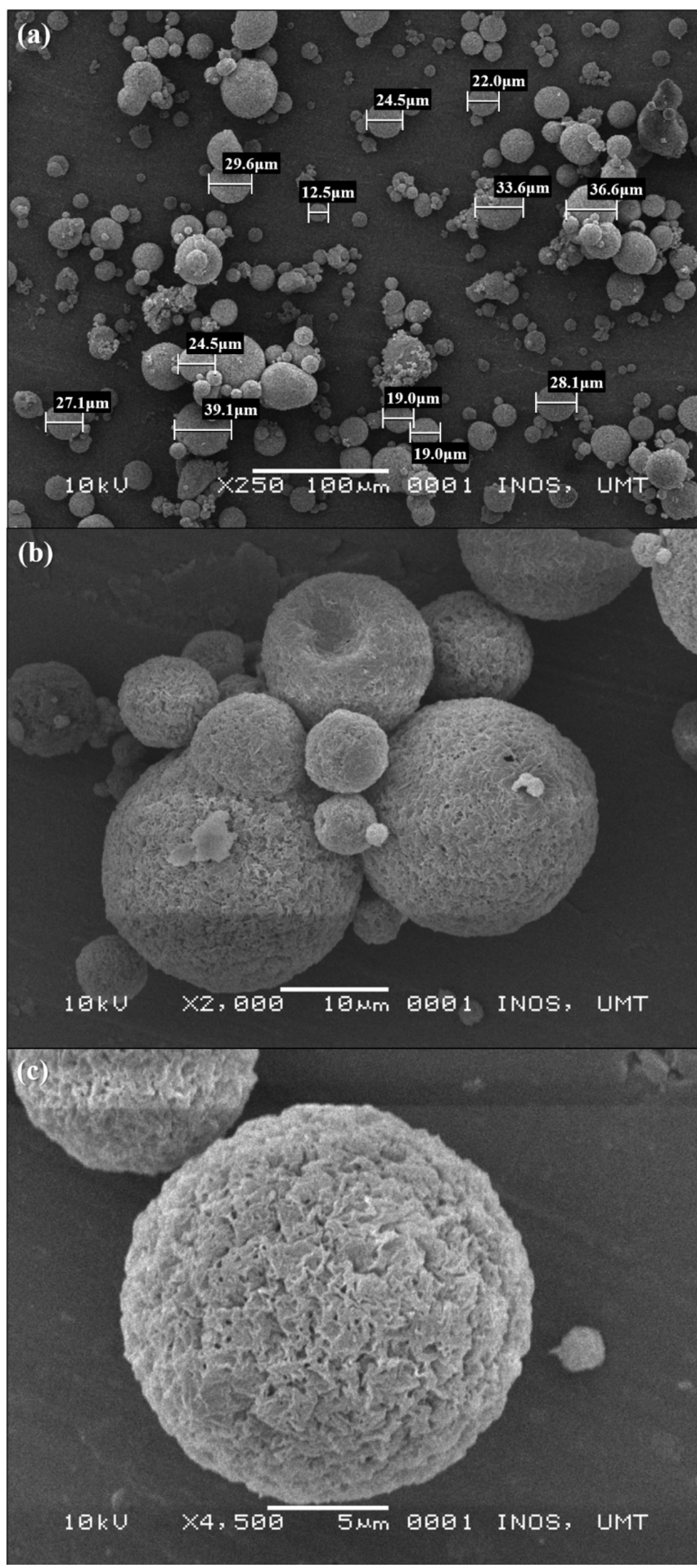

Figure 2: Scanning electron microscope (SEM) images of PHA microbeads with (a) 250x, (b) 2000x and (c) 4500x magnifications 
The Presence, Ingestion and Egestion of $\mathrm{PH} A$ Microbeads by Copepod

The fluorescence of the Nile-red-stained PHA microbeads in $N$. lacustris pacifica confirmed the presence of soluble PHA in the tissue of the living organism. The PHA microbeads were observed in the digestive tracts and faecal pellets of $N$. lacustris pacifica (Figures 3), confirming the ingestion and egestion of the PHA microbeads by the copepods after being exposed for $24 \mathrm{~h}$.
In both filtered and unfiltered seawater treatments, the PHA microbeads ingested by $N$. lacustris pacifica was $>70 \%$ and the egestion time to fully remove the microbeads from the body required only 3 days (Figure 4a). Similarly, the percentage of fluorescent PHA microbeads in faecal pellets followed the same trend of decline from the first day until the third day of exposure (Figure 4b). However, no significant difference on the percentage of PHA microbeads ingested and in faecal pellets were observed between filtered and unfiltered seawater treatment $(p>0.05)$.
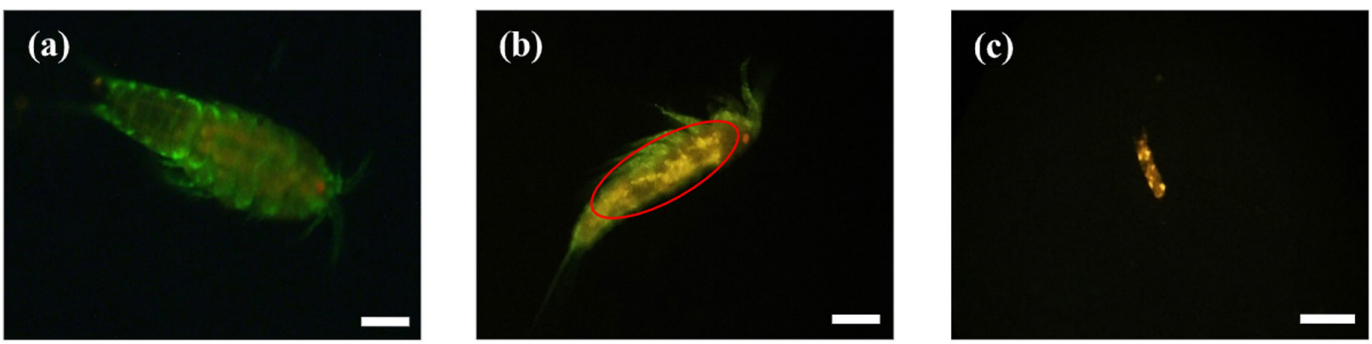

Figure 3: Microscopic images under an inverted fluorescence microscope of $N$. lacustris pacifica; (a) The absence of the PHA microbead in the digestive tract, (b) the presence of the PHA microbead indicated in the red circle, (c) the PHA microbeads in a faecal pellet. [Scale bar: Copepods $=200 \mu \mathrm{m}$; faecal pellet $=100 \mu \mathrm{m}$ ]

(a)

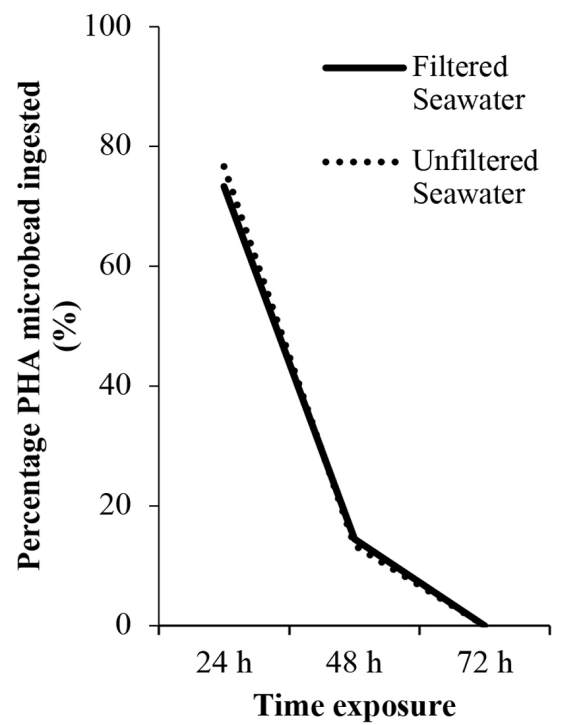

(b)

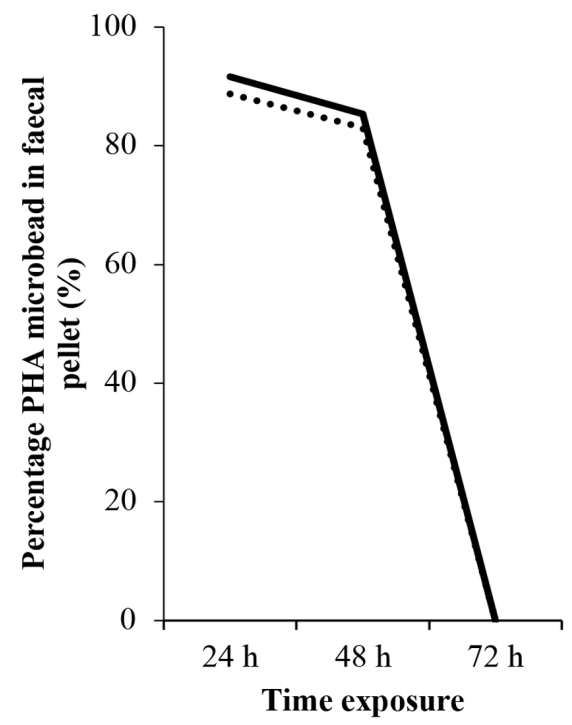

Figure 4: (a) The percentage of PHA microbeads ingested by $N$. lacustris pacifica $(\mathrm{n}=30)$. (b) The percentage of PHA microbeads in the faecal pellets of $N$. lacustris pacifica within three days of exposure. No mortality was observed in this short-term exposure 
Cole et al. (2013) reported a high percentage (>70\%) of microbeads (7000 particle $\mathrm{mL}^{-1} ; 7.3$ $\mu \mathrm{m}$ polystyrene) ingested by copepods Acartia clausi, Calanus helgolandicus, Centropages typicus, and Temora longicornis after an exposure of $24 \mathrm{~h}$. They also demonstrated that dose-dependent microplastic exposure could significantly reduce the algal ingestion rate of the copepod Centropages typicus. Similar results were also observed in copepod Tigriopus japonicus tested using polystyrene beads at a higher concentration $\left(5.25 \times 10^{5}(6 \mu \mathrm{m})-9.1 \times\right.$ $10^{11}$ particle $\left.\mathrm{mL}^{-1}(0.05 \mu \mathrm{m})\right)$ and the presence of beads in faecal pellets was observed after an exposure of $24 \mathrm{~h}$ (Lee et al., 2013). Using a lower particle concentration, the ingestion of polystyrene microbeads $\left(50-200\right.$ particle $\mathrm{mL}^{-}$ $\left.{ }^{1}, 15 \mu \mathrm{m}\right)$ by copepod Calanus finmarchicus was reported to be higher among more than $80 \%$ of the overall individuals after $24 \mathrm{~h}$, while egestion was recorded to be faster than the current study of among $94 \%$ of individuals within $4 \mathrm{~h}$, while the other $6 \%$ of individuals egested faecal pellets within $18 \mathrm{~h}$ (Vroom et al., 2017). Likewise, Dedman (2014) showed that copepod Calanus typicus ingested $100 \%$ of microbeads at a concentration of 100 particles $\mathrm{mL}^{-1}$, whereas $100 \%$ of individuals showed an adherence of microbead to the outer part of the body after an exposure of $24 \mathrm{~h}$. The difference in copepod response after ingestion could be due to species-specific effects, the variations of material and the concentration of microbeads used in the experiment. According to Straub and co-workers (Straub et al., 2017), petroleum-based microplastics have relatively lower ingestion rates, hence a lower assimilation efficiency compared with PHA microplastics, but both microplastic types led to a lower wet weight gain among amphipods. Both microplastic types caused digestive constraints in the amphipods (Straub et al., 2017). Although higher ingestion rates may indicate a better assimilation efficiency, they also lead to a more rapid consumption of microplastic-contaminant complexes in the environment over time.

\section{Conclusion}

Zooplankton N. lacustris pacifica was confirmed to consume PHA microbeads as food, as well as proven to uptake and egest these microbeads with possible assimilation. Data revealed that different seawater treatments did not affect the ingestion and egestion by $N$. lacustris pacifica throughout the analysis. The proven uptake of micro-sized biodegradable plastics by $N$. lacustris pacifica poses new unanswered research questions, such as the potential harm or lack of it when micro-sized biodegradable plastics, such as PHA, polylactic acid (PLA), starch blends and cellulose-based plastics, are consumed by zooplankton. The chronic exposure of microplastics to several copepod generations, with selected range of microbead size and concentration, are needed to study the effect of biodegradable microplastics on copepod mortality.

\section{Acknowledgements}

The authors are thankful for the funding from Postgraduate Research Grant, Universiti Malaysia Terengganu (55193/1).

\section{References}

Amelia, T. S. M., Govindasamy, S., Tamothran, A. M., Vigneswari, S., \& Bhubalan, K. (2019). Applications of PHA in Agriculture. In Kalia, V. C. (Ed.), Biotechnological Applications of Polyhydroxyalkanoates (pp. 347-361). Singapore: Springer.

Amirul, A. A., Syairah, S. N., Ahmad, R. M. Y., Azizan, M. N. M., \& Majid, M. I. A. (2008). Synthesis of biodegradable polyesters by Gram negative bacterium isolated from Malaysian environment. World Journal of Microbiology \& Biotechnology, 24(8), 1327-1332. doi: 10.1007/s11274-0079605-2.

Auta, H. S., Emenike, C. U., \& Fauziah, S. H. (2017). Distribution and importance of microplastics in the marine environment: 
A review of the sources, fate, effects, and potential solutions. Environment International, 102, 165-176.

Avio, C. G., Gorbi, S., Milan, M., Benedetti, M., Fattorini, D., d'Errico, G., Pauletto, M., Bargelloni, L., \& Regoli, F. (2016). Pollutants bioavailability and toxicological risk from microplastics to mussels. Environmental Pollution, 198, 211-222.

Barnes, D. K. A., Galgani, F., Thompson, R. C., \& Barlaz, M. (2009). Accumulation and fragmentation of plastic debris in global environments. Philosophical Transactions of the Royal Society B, 364(1526), 19851998.

Bejgarn, S., MacLeod, M., Bogdal, C., \& Breitholtz, M. (2015). Toxicity of leachate from weathering plastics: An exploratory screening study with Nitocra spinipes. Chemosphere, 132, 114-119.

Carr, S. A., Liu, J., \& Tesoro, A. G. (2016). Transport and fate of microplastic particles in wastewater treatment plants. Water Research, 91, 174-182.

Cerrone, F., Sánchez-Peinado, M. D. M., Rodríguez-Díaz, M., González-López, J., \& Pozo, C. (2011). PHAs production by strains belonging to Massilia genus from starch. Starch/Staerke, 63, 236-240.

Cole, M., Lindeque, P., Fileman, E., Halsband, C., Goodhead, R., Moger, J., \& Galloway, T. S. (2013). Microplastic ingestion by zooplankton. Environmental Science and Technology, 47(12), 6646-6655.

Cole, M., \& Galloway, T.S. (2015). Ingestion of nanoplastics and microplastics by Pacific oyster larvae. Environmental Science and Technology, 49, 14625e14632. doi: 10.1021/acs.est.5b04099.

Coppock, R. L., Galloway, T. S., Cole, M., Fileman, E. S., Queirós, A. M., \& Lindeque, P. K. (2019). Microplastics alter feeding selectivity and faecal density in the copepod, Calanus helgolandicus. Science of the Total Environment, 687, 780-789.
Dedman, C. J. (2014). Investigating Microplastic Ingestion by Zooplankton. $\mathrm{PhD}$ Thesis, Exeter University, England, 1-144.

Draelos, Z. D. (2019). Cosmeceuticals: What's real, what's not. Dermatologic Clinics, 37,107-115.

Flament, F., Francois, G., Qiu, H., Ye, C., Hanaya, T., Batisse, D., CointereauChardon, S., Seixas, M. D. G., Belo, S. E. D., \& Bazin, R. (2015). Facial skin pores: A multi-ethnic study. Clinical, Cosmetic and Investigational Dermatology, 8, 85-93.

Govindasamy, S., Syafiq, I. M., Amirul, A. A., Amin, R. M., \& Bhubalan, K. (2019). Dataset on controlled production of polyhydroxyalkanoate-based microbead using double emulsion solvent evaporation technique. Data in Brief, 23, 103675. doi: 10.1016/j.dib.2019.01.023.

Han, X., Satoh, Y., Kuriki, Y., Seino, T., Fujita, S., Suda, T., Kobayashi, T., \& Tajima, K. (2014). Polyhydroxyalkanoate production by a novel bacterium Massilia sp. UMI21 isolated from seaweed, and molecular cloning of its polyhydroxyalkanoate synthase gene. Journal of Bioscience and Bioengineering, 118, 514-519.

Isobe, A. (2016). Percentage of microbeads in pelagic microplastics within Japanese coastal waters. Marine Pollution Bulletin, 110, 432-437.

Kiun, J. T., Amelia, T. S. M., Huong, K. H., Amirul, A. A., \& Bhubalan, K. (2019). Optimizing the biosynthesis of renewable polyhydroxyalkanoate copolymer containing 3-hydroxyvalerate by Massilia haematophila using statistical modelling. BioTechnologia, 100(4), 359-371.

Kiun, J. T., Bhubalan, K., \& Amirul, A. A. (2016). Novel PHA bioplastic producing bacteria isolated from brackish environment, presented at the 14th Symposium of Malaysian Society of Applied Biology, Melaka, 29-31 May 2016. 
Lee, K. W., Shim, W. J., Kwon, O. Y., \& Kang, J. H. (2013). Size-dependent effects of micro polystyrene particles in the marine copepod tigriopus japonicus. Environmental Science and Technology, 47(19), 11278-11283.

Md Amin, R., Sohaimi E.S., Anuar, S.t., \& Bachok, Z. (2020). Microplastic ingestion by zooplankton in Terengganu coastal waters, southern South China Sea. Marine Pollution Bulletin, 150, 110616. https://doi. org/10.1016/j.marpolbul.2019.110616

Mohamed, S., Amirul, A. A., Effendy, A. W. M., \& Bhubalan, K. (2017). Characterization and cytotoxicity of polyhydroxyalkanoate microparticles as adjuvant matrix for the immobilization of Pasteurella multocida whole-cell vaccine. Journal of Sustainability Science and Management, 12(2), 89-95.

Murueva, A., Shishatskaya, E., Kuzmina, A., Volova, T., \& Sinskey, A. (2013). Microparticles prepared from biodegradable polyhydroxyalkanoates as matrix for encapsulation of cytostatic drug. Journal of Materials Science: Materials in Medicine, 24(8), 1905-1915.

Napper, I. E., Bakir, A., Rowland, S. J., \& Thompson, R. C. (2015). Characterisation, quantity and sorptive properties of microplastics extracted from cosmetics. Marine Pollution Bulletin, 99(1-2), 178185.

Ntaikou, I., Koumelis, I., Tsitsilianis, C., Parthenios, J., \& Lyberatos, G. (2018). Comparison of yields and properties of microbial polyhydroxyalkanoates generated from waste glycerol based substrates. International Journal of Biological Macromolecules, 112, 273-283.

Rao, U., Kumar, R., Balaji, S., \& Sehgal, P. K. (2010). A novel biocompatible poly(3hydroxy-co-4-hydroxybutyrate) blend as a potential biomaterial for tissue engineering. Journal of Bioactive and Compatible Polymers, 25(4), 419-436.

Straub, S., Hirsch, P. E., \& Burkhardt-Holm, P. (2017). Biodegradable and petroleum-based microplastics do not differ in their ingestion and excretion but in their biological effects in a freshwater invertebrate Gammarus fossarum. International Journal of Environmental Research and Public Health, 14, 774. doi: 10.3390/ijerph14070774.

Tanaka, K., \& Takada, H. (2016). Microplastic fragments and microbeads in digestive tracts of planktivorous fish from urban coastal waters. Scientific Reports, 6, 34351.

Turner, J. T. (2004). The importance of small pelagic planktonic copepods and their role in pelagic marine food webs. Zoological Studies, 43, 255-266.

Verlinden, R., Hill, D., Kenward, M., Williams, C., \& Radecka, I. (2007). Bacterial synthesis of biodegradable polyhydroxyalkanoates. Journal of Applied Microbiology, 102(6), 1437-1449.

Vroom, R. J. E., Koelmans, A. A., Besseling, E., \& Halsband, C. (2017). Aging of microplastics promotes their ingestion by marine zooplankton. Environmental Pollution, 231(1), 987-996.

Williams, S. F., \& Martin, D. P. (2002). Polyesters III: Applications and commercial products. In Y. Doi \& A. Steinbüchel (Eds.), Biopolymers. WILEY-VCH. Germany: pp. 91-127.

Yatim, A., Syafiq, I., Huong, K., Amirul, A., Effendy, A., \& Bhubalan, K. (2017). Bioconversion of novel and renewable agroindustry by-products into a biodegradable poly(3-hydroxybutyrate) by marine Bacillus megaterium UMTKB-1 strain. BioTechnologia, 98(2), 141-151. 